\title{
Development of reference standard for small angle scattering measurement q calibration
}

\author{
R. Joseph Kline \\ National Institute of Standards and Technology, Gaithersburg, MD, USA \\ joe.kline@nist.gov
}

We report on the development of a reference standard for q calibration of small angle scattering measurements. The standard consists of a $100 \mathrm{~nm}$ pitch line grating on a silicon nitride membrane. The grating is $100 \mathrm{~nm}$ tall and $40 \mathrm{~nm}$ wide tungsten lines. Tungsten was selected to give strong scattering intensity while not having absorption features around the carbon k-edge. The silicon nitride membrane allows measurements over a large range of beam energies. The test structure has a $1 \mu \mathrm{m}$ two-dimensional grating superimposed on the $100 \mathrm{~nm}$ line grating. The superlattice provides additional scattering peaks that can only be resolved in high-resolution configurations. The test structure allows evaluation of q-resolution in addition to calibration of q.

The prototype structure was tested between $250 \mathrm{eV}$ and $24.5 \mathrm{keV}$ and provided strong scattering at all energies. Figure 1 shows an example scattering pattern collected in a $60 \mathrm{~s}$ exposure on a laboratory SAXS system using Ga Kalpha. The scattering pattern allows calibration between $0.0006 \AA^{-1}$ and $0.1 \AA^{-1}$. We will also discuss measurements made at SAXS beamlines and at soft X-ray scattering beamlines.

a)

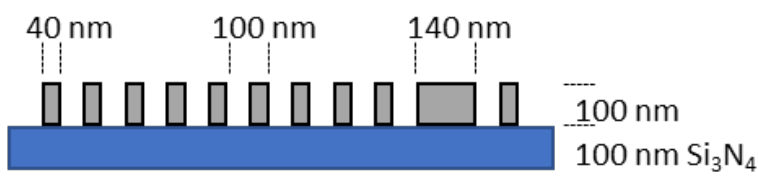

b)

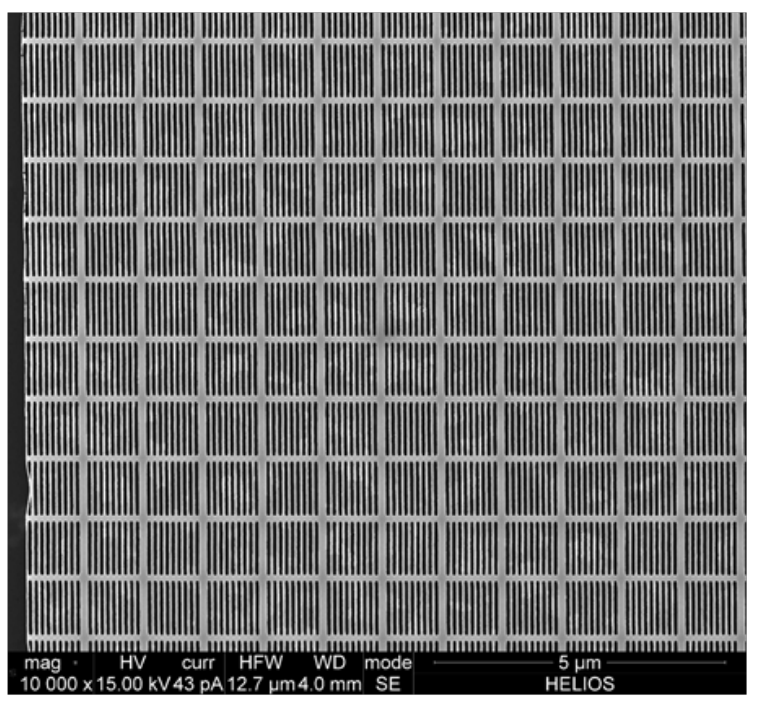

c)

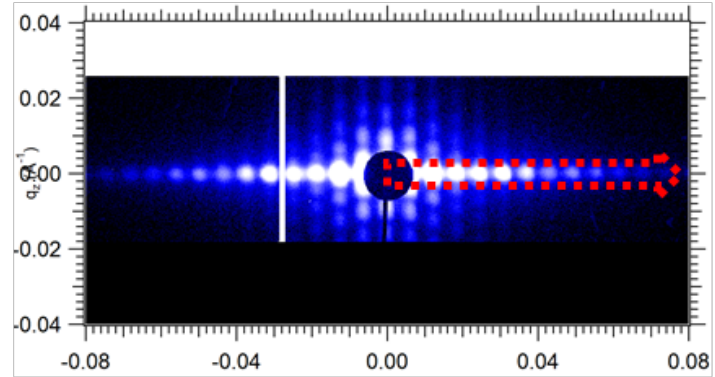

d)

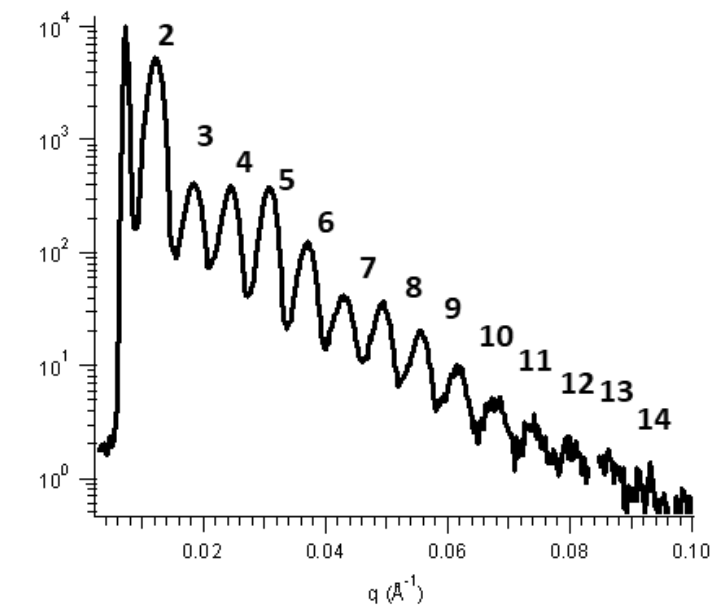

Figure 1. a) Schematic of tungsten test structure, b) SEM image of structure, c) lab SAXS pattern in $60 \mathrm{~s}$, d) 1D integration .

Keywords: Small angle X-ray scattering; calibration; calibration standard 